\title{
Evaluation of the status of use of chemicals and antibiotics in freshwater aquaculture activities with special emphasis to fish health management
}

\author{
M.A.R. Faruk, M.M. Ali and Z.P. Patwary \\ Department of Aquaculture, Bangladesh Agricultural University, Mymensingh-2202, Bangladesh
}

\begin{abstract}
The study was carried out to understand current status of use of chemicals and antibiotics in fish health management in freshwater aquaculture activities. Data were collected through questionnaire interview, personal contact, market survey and participatory rural appraisal like focus group discussion with fish and prawn farmers, retailers of animal medicine and representatives of pharmaceutical companies. A range of chemicals including antibiotics were found available in market. Fish health management and disease treatment were the major areas where majority of such chemicals have been used. Other uses included pond preparation and management, growth promotion and improvement of water quality to enhance pond productivity. Commonly found traditional chemicals in health management included Lime, Salt, Potassium permanganate, Sumithion, Melathion, Formalin, Bleaching powder and Malachite green. Of the new products JVzeolite, Geotox, Green zeolite, Orgavit aqua, Fish vitaplus, AQ grow-G, Oxy flow, Oxy max and $\mathrm{O}_{2}$-marine were most widely used compounds. Fourteen branded antibiotics were found in market of which Oxysentin, Renamox, Renamycin and Orgamycine were being widely used. Thirty three pharmaceutical companies were producing and marketing these products. The study also identified the problems associated with the use of chemicals which included lack of knowledge regarding use of chemicals, appropriate dose, method of application and indiscriminate use of chemicals.
\end{abstract}

Keywords: Chemicals, Antibiotics, Aquaculture, Health management

\section{Introduction}

Aquaculture in Bangladesh is expanding rapidly. Over the last decade it has expanded, diversified, intensified and technologically advanced. In aquaculture, as in all food production sectors, one of the inputs required for successful fish production is chemical. Chemicals are indeed an essential ingredient to successful aquaculture, which has been used in various forms for centuries (Subasinghe et al., 1996). Chemicals used in aquaculture can be classified as purpose of use, the type of organisms under culture, the life cycle stage for which they are used, the culture system and intensity of culture, and the type of people who are using them. Chemicals and antibiotics are important components in health management of aquatic animals, pond construction, soil and water management, improve aquatic productivity, transportation of live fish, feed formulation, manipulation of reproduction, growth promotion and processing and value addition of the final product (GESAMP, 1997; Subasinghe et al., 1996). A variety of other chemicals are also used in aquaculture for health management of fish apart from antibiotics. Some common chemicals include sodium chloride, formalin, malachite green, methylene blue, potassium permanganate, hydrogen per oxide, copper compounds, glutaraldehyde and trifluralin (Plumb, 1992).

A range of disease could be found in farmed aquatic animals in Bangladesh (Karim and Stellwagen, 1998; BFRI, 1999 and Faruk et al., 2004). Farmers are using a range of chemicals and antibiotic for the treatment of diseased animal. Also, pharmaceutical companies and chemical sellers are influencing fish and prawn farmers to buy their products. The farmers have been using these chemicals without knowing their necessity and effectiveness. 
There are problems associated with the use of chemicals. With the expansion of aquaculture in Bangladesh, there has been increasing trend in using chemicals in aquatic animal health management. Commonly used chemicals in Bangladesh aquaculture are lime, rotenone, various forms of inorganic and organic fertilizers, phostoxin, salt, dipterex, antimicrobials, potassium permanganate, copper sulphate, formalin, sumithion, melathion etc. (Phillips, 1996; Hasan and Ahmed, 2002; Brown and Brooks, 2002; DoF, 2002 and Faruk et al., 2005).

Unfortunately, little attention has been paid on the documentation of chemicals and antibiotics used in aquaculture industry in the country. As a result, there is a lack of information regarding the present status and consequences of chemicals and antibiotics using in aquaculture sector especially in aquatic animal health management and needs examination. Hence, the objectives of the present study were i) to identify types of chemical and antibiotic used in aquatic animal health management; ii) to know the purpose, dose and methods of their application and iii) to identify the problems associated with their use.

\section{Materials and Methods}

The study focused mainly on different upazillas in Mymensingh district where aquaculture clusters are located. Data were collected through questionnaire interview, personal contact, market survey and participatory rural appraisal like focus group discussion with fish and prawn farmers, retailers of animal medicine and representatives of pharmaceutical companies. For questionnaire survey a set of preliminary questionnaire based on the objectives of the study was prepared. The preliminary questionnaire was tested twice at the field level with few farmers and based on the response, the final set questionnaire was prepared. For the interview, simple random sampling method was followed.

PRA tools such as focus group discussion were conducted with rural fish farmers. Six to twelve fish farmers were selected for each discussion. Key informants such as District Fisheries Officer, Upazilla Fisheries Officer and NGO workers were interviewed by a semistructured questionnaire. Key issues that came from focus group discussion were also discussed with key informants to have more logical explanation.

\section{Results and Discussion}

The chemicals available in the market used at different stages of aquatic animal health management like pond preparation, growth promotion, increasing oxygen concentration, disinfectant, probiotic and fish and prawn disease treatment have been collected and compiled. Fish disease treatment was the major area where plenty of such compounds were used. The local animal feed and chemical shops are the main sources of such compounds.

\section{Chemicals used for pond preparation and water quality management}

A number of traditional as well as new compounds were seen in the market which are being used to pond preparation and improving water quality of fish pond. The list of such chemicals with their active ingredients, prescribed dose, source and approximate price are shown in Table 1.

Chemicals like Rotenone and Predator are used for controlling unwanted fishes as well as other harmful aquatic animals. Fertilizers are used to increase primary productivity. Controller and Plankto Forte are used as a good source of mineral. 
Faruk et al.

Table 1. Chemicals used for pond preparation and water quality management

\begin{tabular}{|c|c|c|c|c|}
\hline Trade Name & Active ingredient & Dose & Source & Price (Tk.) \\
\hline Geotox & $\begin{array}{l}\mathrm{SiO}_{2}, \mathrm{Al}_{2} \mathrm{O}_{3}, \mathrm{Fe}_{2} \mathrm{O}_{3} \mathrm{CaO} \\
\mathrm{MgO}, \mathrm{Na}_{2} \mathrm{O}\end{array}$ & $\begin{array}{l}\text { For 3-6 feet deep water body } 20-25 \mathrm{Kg} \\
/ 100 \text { dec. After stocking } 10-20 \mathrm{~kg} / 100 \\
\text { dec. }\end{array}$ & \begin{tabular}{|l} 
Novartis \\
Pharmaceuticals
\end{tabular} & $55 / \mathrm{kg}$ \\
\hline JV Zeolite & $\begin{array}{l}\mathrm{SiO}_{2}, \mathrm{Al}_{2} \mathrm{O}_{3}, \mathrm{Fe}_{2} \mathrm{O}_{3} \mathrm{CaO} \\
\mathrm{MgO}, \mathrm{Na}_{2} \mathrm{O}, \mathrm{K}_{2} \mathrm{O}\end{array}$ & $\begin{array}{l}\text { During pond preparation } 7 \mathrm{~kg} / 33 \mathrm{dec} \text {. } \\
\text { During culture } 3.5 \mathrm{Kg} / 33 \mathrm{dec} \text {. every } 15 \\
\text { days }\end{array}$ & $\begin{array}{l}\text { Eon Animal } \\
\text { health Products }\end{array}$ & $50 / \mathrm{kg}$ \\
\hline Green Zeolite & $\begin{array}{l}\mathrm{SiO}_{2}, \mathrm{Al}_{2} \mathrm{O}_{3}, \mathrm{Fe}_{2} \mathrm{O}_{3} \mathrm{CaO}, \\
\mathrm{MgO}, \mathrm{Na}_{2} \mathrm{O}, \mathrm{K}_{2} \mathrm{O}\end{array}$ & $\begin{array}{l}\text { During pond preparation } 20-25 \mathrm{Kg} / 100 \\
\text { dec. During culture } 10-15 \mathrm{~kg} / 100 \mathrm{dec} \text {. }\end{array}$ & \begin{tabular}{|l} 
Organic \\
Pharmaceuticals
\end{tabular} & $55 / \mathrm{kg}$ \\
\hline Pontox plus & $\begin{array}{l}\mathrm{SiO}_{2}, \mathrm{Al}_{2} \mathrm{O}_{3}, \mathrm{Fe}_{2} \mathrm{O}_{3} \mathrm{CaO}, \\
\mathrm{MgO}, \mathrm{Na}_{2} \mathrm{O}\end{array}$ & $\begin{array}{l}\text { For 3-6 feet deep water body } 15 \\
\mathrm{~kg} / 100 \text { dec. After stocking } 10-20 \mathrm{~kg} \text {. }\end{array}$ & Rals Agro Ltd. & $55 / \mathrm{kg}$ \\
\hline Zeolite & $\begin{array}{l}\mathrm{SiO}_{2}, \mathrm{Al}_{2} \mathrm{O}_{3}, \mathrm{Fe}_{2} \mathrm{O}_{3} \mathrm{CaO}, \\
\mathrm{MgO}, \mathrm{Na}_{2} \mathrm{O}\end{array}$ & $20-30 \mathrm{~kg} / \mathrm{acre}$ & $\begin{array}{l}\text { National Agricare } \\
\text { Imp. Exp Ltd }\end{array}$ & $50 / \mathrm{kg}$ \\
\hline Benzo & $\begin{array}{l}\text { Tetra acetyl ethylin } \\
\text { diamin + Sodium } \\
\text { perborate }\end{array}$ & $200 \mathrm{gm} / \mathrm{acre}$ & Synzenta & $400 / 250 \mathrm{~g}$ \\
\hline Zeocare & $\begin{array}{l}\mathrm{SiO}_{2}, \mathrm{Al}_{2} \mathrm{O}_{3}, \mathrm{Fe}_{2} \mathrm{O}_{3} \mathrm{CaO}, \\
\mathrm{MgO}, \mathrm{Na}_{2} \mathrm{O}\end{array}$ & $20-30 \mathrm{~kg} / \mathrm{acre}$ & Nature Care & $50 / \mathrm{kg}$ \\
\hline Mega Zeo & $\begin{array}{l}\mathrm{SiO}_{2}, \mathrm{Al}_{2} \mathrm{O}_{3}, \mathrm{Fe}_{2} \mathrm{O}_{3} \mathrm{CaO} \\
\mathrm{MgO}, \mathrm{Na}_{2} \mathrm{O}, \mathrm{K}_{2} \mathrm{O}, \mathrm{Mn}\end{array}$ & $\begin{array}{l}\text { For } 3-6 \text { feet deep water body } 25 \mathrm{~kg} \\
/ 100 \text { dec. After stocking } 15-20 \mathrm{~kg} \text { in } \\
\text { same water body. }\end{array}$ & $\begin{array}{l}\text { ACl Animal } \\
\text { Health }\end{array}$ & $45 / \mathrm{kg}$ \\
\hline Lime & $\mathrm{CaO}, \mathrm{Ca}(\mathrm{OH})_{2}$ & $1-2 \mathrm{~kg} / \mathrm{dec}$ & Chemical seller & $12 / \mathrm{kg}$ \\
\hline Agriculturelime & $\mathrm{CaCO}_{3}$ & $\begin{array}{l}\text { Spread with water } \\
6-10 \mathrm{ppm}\end{array}$ & Chemical seller & $8-15 / \mathrm{kg}$ \\
\hline Bis Zeolite & $\begin{array}{l}\mathrm{SiO}_{2}, \mathrm{Al}_{2} \mathrm{O}_{3}, \mathrm{Fe}_{2} \mathrm{O}_{3} \mathrm{CaO}, \\
\mathrm{MgO}, \mathrm{Na}_{2} \mathrm{O} \\
\end{array}$ & 20-30Kg/acre & $\begin{array}{l}\text { Avon Animal } \\
\text { Health } \\
\end{array}$ & $50 / \mathrm{kg}$ \\
\hline SupureZeolite & $\begin{array}{l}\mathrm{SiO}_{2}, \mathrm{Al}_{2} \mathrm{O}_{3}, \mathrm{Fe}_{2} \mathrm{O}_{3} \mathrm{CaO}, \\
\mathrm{MgO}, \mathrm{Na}_{2} \mathrm{O}\end{array}$ & $20-30 \mathrm{~kg} /$ acre & $\begin{array}{l}\text { Avon Animal } \\
\text { Health }\end{array}$ & $50 / \mathrm{kg}$ \\
\hline Bio-Tuff & $\begin{array}{l}\mathrm{SiO}_{2}, \mathrm{Al}_{2} \mathrm{O}_{3}, \mathrm{Fe}_{2} \mathrm{O}_{3} \mathrm{CaO} \\
\mathrm{MgO}, \mathrm{Na}_{2} \mathrm{O}, \mathrm{K}_{2} \mathrm{O}, \mathrm{TiO}_{2}\end{array}$ & $\begin{array}{l}\text { During pond preparation } 15-20 \mathrm{~kg} / 100 \\
\text { dec. During culture } 7-10 \mathrm{~kg} / 100 \mathrm{dec} . \\
\text { Every } 30-40 \text { days. }\end{array}$ & \begin{tabular}{|l|} 
Organic \\
Pharmaceuticals \\
Ltd. \\
\end{tabular} & $50 / \mathrm{kg}$ \\
\hline Well Zeolite & $\begin{array}{l}\mathrm{SiO}_{2}, \mathrm{Al}_{2} \mathrm{O}_{3}, \mathrm{Fe}_{2} \mathrm{O}_{3} \mathrm{CaO} \\
\mathrm{MgO}, \mathrm{Na}_{2} \mathrm{O}\end{array}$ & $\begin{array}{l}\text { For 3-6 feet deep water body } 6-8 \mathrm{~kg} \\
\text { /33dec dec. After stocking 3-6 kg }\end{array}$ & $\mathrm{SK}+\mathrm{F}$ & $50 / \mathrm{kg}$ \\
\hline Bio Aqua & $\begin{array}{l}\text { Extract of Uka cidizera } \\
\text { tree }\end{array}$ & $2 \mathrm{ml} / 100 \mathrm{dec}$ (1m depth) & \begin{tabular}{|l|} 
Eon Animal \\
health Products
\end{tabular} & $\begin{array}{l}330 / 100 \\
\mathrm{ml}\end{array}$ \\
\hline Aquazet & $\begin{array}{l}\mathrm{SiO}_{2}, \mathrm{Al}_{2} \mathrm{O}_{3}, \mathrm{Fe}_{2} \mathrm{O}_{3} \mathrm{CaO}, \\
\mathrm{MgO}, \mathrm{Na}_{2} \mathrm{O}\end{array}$ & 20-30/acre & Lion Overseas & $50 / \mathrm{kg}$ \\
\hline
\end{tabular}

\section{Chemicals use as disinfectant}

Disinfectants are widely used in many spheres of aquaculture. They are used both in hatchery and grow-out systems mainly for equipment cleaning, to maintain hygiene and in some cases to treat disease. The disinfectants listed in Table 2 were found available in the market.

According to the leaflet provided by the company, Timsen and Emsen are also very effective in prevention of some bacterial and fungal infection as well as they destroy viruses. Formalin is also used to control protozoan disease. BKC (Benzal Konium Chloride) used for controlling bacterial disease and Efinol can also be used as stress resistance.

\section{Chemicals used to increase oxygen concentration}

Several chemicals were seen readily available in the chemical shops to use for increasing dissolved oxygen in fish pond. Oxydizing agent, hydrogen peroxide are the major active ingredients of such chemical (Table 3). 
Table 2. Chemicals used as disinfectant

\begin{tabular}{|c|c|c|c|c|}
\hline Trade Name & Active ingredient & Dose & Source & Price (Tk.) \\
\hline EDTA & Sodium thio sulfate & $0.1-1 \mathrm{ppm}$ & Chemical seller & $40 / \mathrm{kg}$ \\
\hline Bleaching & Chlorine & $60 \mathrm{ppm}$ & Chemical seller & $50 / \mathrm{kg}$ \\
\hline Timsen & $\begin{array}{l}\text { n-alkyl dimethyl benzyl ammonium chloride+ } \\
\text { stabilized urea }\end{array}$ & $\begin{array}{l}20 \mathrm{~g} / 33 \mathrm{dec} \text {. (For prevention) } \\
80 \mathrm{~g} / 33 \mathrm{dec} \text {. (For Treatment) }\end{array}$ & $\begin{array}{l}\text { Eon Animal Health Products } \\
\text { Ltd. }\end{array}$ & $238 / 50 \mathrm{~g}$ \\
\hline Emsen & $\begin{array}{l}\text { n-alkyl dimethyl benzyl ammonium chloride+ } \\
\text { stabilized urea }\end{array}$ & $80 \mathrm{~g} / 33 \mathrm{dec}$ & $\begin{array}{l}\text { SK+FEskayef Bangladesh } \\
\text { Ltd. }\end{array}$ & $238 / 50 \mathrm{~g}$ \\
\hline Water clear & Sodium Thiosulphate & $\begin{array}{l}\text { In case of 5-6 feet deep } \\
\text { water body } 2-3 \mathrm{~L} / 100 \mathrm{dec} \text {. }\end{array}$ & Organic Pharmaceuticals Ltd. & $300 / L$ \\
\hline Pathocide & $\begin{array}{l}\text { Benzyl chronium + Natural polymer of } \\
\text { glucosamine }\end{array}$ & $200 \mathrm{ml} / 33 \mathrm{dec}$ & Penta Agrovet Ltd & $100 / 100 \mathrm{ml}$ \\
\hline Omicide & Benzyl ammonium chloride+ urea & $\begin{array}{l}200 \mathrm{ml} / 33 d e c . \text { After } 24 \text { hours } \\
150 \mathrm{ml}\end{array}$ & $\begin{array}{l}\text { Lion OverseasTrading } \\
\text { Company }\end{array}$ & $100 / 100 \mathrm{ml}$ \\
\hline Lenocide & $\begin{array}{l}\text { Alkyl benzyl } \\
\text { dimethyl ammonium chloride+Poly-2-deoxy-2 } \\
\text { Amino glucose }\end{array}$ & $500-1000 \mathrm{ml} / \mathrm{acre}$ & Nature Care Com. & $100 / 100 \mathrm{ml}$ \\
\hline $\begin{array}{l}\text { Microdine- } \\
\text { lodine } 20 \% \\
\end{array}$ & $\begin{array}{l}\text { Nonyl alkl ohenoxy poly ethane oxide iodine } \\
\text { complex }\end{array}$ & 2-2.5 L/ccre & Rals Agro Ltd. & $80 / 400 \mathrm{~g}$ \\
\hline Formalin & $38 \%$ formaldehyde & $1-3 \mathrm{ppm}$ & Chemical seller & $70 / \mathrm{kg}$ \\
\hline $\mathrm{BKC}$ & Benzal Konium chloride & Spread with water, $0.5 \mathrm{ppm}$ & Chemical seller & $\begin{array}{l}\text { Tk.1000- } \\
1500 / \mathrm{Kg}\end{array}$ \\
\hline Efinol & Efinol & $\begin{array}{l}\text { 5-8 g/1000 Liter } \\
\text { water }\end{array}$ & $\begin{array}{l}\text { Eon Animal health Products } \\
\text { Ltd. }\end{array}$ & - \\
\hline
\end{tabular}

Table 3. Chemicals used for oxygen supply

\begin{tabular}{|c|c|c|c|c|}
\hline Trade Name & Active ingredient & Dose & Source & Price (Tk.) \\
\hline Oxyflow & $\mathrm{H}_{2} \mathrm{O}_{2} 10 \%$ & $\begin{array}{l}\text { General dose } 250-350 \text { g/acre } \\
\text { In case of high deficiency } 500 \text { gm/acre in } \\
\text { same water body. }\end{array}$ & Novartis Pharmaceuticals Ltd. & $600 / \mathrm{kg}$ \\
\hline Oxymax & $\mathrm{H}_{2} \mathrm{O}_{2} 10 \%$ & 250-500gm/acre (1 m deep water body) & Eon Animal health Products Ltd. & $600 / \mathrm{kg}$ \\
\hline $\mathrm{O}_{2}$-Marine & $\mathrm{H}_{2} \mathrm{O}_{2} 10 \%$ & $\begin{array}{l}\text { 33-40 Tab./33dec. (Generally) } \\
\text { 66-90Tab/33dec }\end{array}$ & Organic Pharmaceuticals Ltd. & $530 / \mathrm{kg}$ \\
\hline Bio care & Sodium Lorile Ether Sulphate & $80-120 \mathrm{ml} / 100 \mathrm{dec}$. & $\mathrm{ACl}$ Animal Health & $350 / \mathrm{L}$ \\
\hline Bio- Ox & Sodium carbonat, $\mathrm{H}_{2} \mathrm{O}_{2}$ & $\begin{array}{l}\text { General dose } 2.5-5.0 \text { g/acre } \\
\text { In case of high deficiency } 5-8 \text { g/acre in same } \\
\text { water body. }\end{array}$ & $\mathrm{ACl}$ Animal Health & $500 / \mathrm{kg}$ \\
\hline Oxy plus & $\begin{array}{l}\mathrm{Na}_{2} \mathrm{O}_{2+} \mathrm{AlOH} \\
\mathrm{Na}_{2} \mathrm{O}_{2}-90 \%\end{array}$ & $500 \mathrm{~g} / \mathrm{acre}$ & Navana Animal Health & $530 / \mathrm{kg}$ \\
\hline Oxygen Plus & $\begin{array}{l}\mathrm{O}_{2} \text { Promoter } \\
\left(\mathrm{H}_{2} \mathrm{O}_{2} / \mathrm{Ca}_{2} \mathrm{O}_{2}\right)\end{array}$ & $\begin{array}{l}\text { General dose } 250-500 \text { g/acre; } \\
\text { In case of high deficiency } 750-1000 \text { gm/acre }\end{array}$ & Avon Animal Health & $530 / \mathrm{kg}$ \\
\hline Oxymore & $\begin{array}{l}\text { Sodium carbonat } \\
\text { peroxyhydrat }\end{array}$ & $\begin{array}{l}\text { General dose } 250-500 \text { g/acre; } \\
\text { In case of high deficiency } 750-1000 \text { gm/acre }\end{array}$ & SK+F Bangladesh Ltd. & $500 / \mathrm{kg}$ \\
\hline Quick Oxygen & $\begin{array}{l}\text { Sodium per carbonat+free } \\
\text { Oxygen }\end{array}$ & $\begin{array}{l}\text { General dose } 250-350 \text { g/acre; } \\
\text { In case of high deficiency } 500 \text { g/acre }\end{array}$ & Organic Pharmaceuticals Ltd. & $600 / \mathrm{kg}$ \\
\hline Oxy-Plus & $\begin{array}{l}\mathrm{O}_{2} \text { Promoter } \\
\left(\mathrm{H}_{2} \mathrm{O}_{2} / \mathrm{Ca}_{2} \mathrm{O}_{2}\right)\end{array}$ & 500 g/acre & Penta Agrovet Ltd & $530 / \mathrm{kg}$ \\
\hline O-Plus & $\begin{array}{l}\mathrm{O}_{2} \text { Promoter } \\
\left(\mathrm{H}_{2} \mathrm{O}_{2} / \mathrm{Ca}_{2} \mathrm{O}_{2}\right)\end{array}$ & 500 g/acre & Nature Care & $500 / \mathrm{kg}$ \\
\hline Oxygrow & $\begin{array}{l}\mathrm{O}_{2} \text { Promoter } \\
\left(\mathrm{H}_{2} \mathrm{O}_{2} / \mathrm{Ca}_{2} \mathrm{O}_{2}\right)\end{array}$ & 500 g/acre & Century Agro Ltd & $530 / \mathrm{kg}$ \\
\hline Oxysun & $\begin{array}{l}\text { Sodium peroxide, Calcium } \\
\text { Peroxide, Magnesium Oxide, } \\
\text { Zinc Oxide }\end{array}$ & 500 g/acre & Rals Agro Ltd. Bangladesh & $530 / 5 \mathrm{~kg}$ \\
\hline $\begin{array}{l}\text { Fish care } \\
\text { powder }\end{array}$ & $\begin{array}{l}\text { Oxide of } \mathrm{Ca}, \mathrm{P}, \mathrm{S}, \mathrm{Mn}, \\
\mathrm{Mg}, \mathrm{Cu}, \mathrm{N} .\end{array}$ & $1 \mathrm{~kg} / 33 \mathrm{dec}$. & S.S.S. Agro Care Ltd & $70 / 400 \mathrm{~g}$ \\
\hline Fish Curepas & $\begin{array}{l}\text { Oxide of Ca,P,S,Mn, } \\
\text { Mg,Cu,N. }\end{array}$ & $1 \mathrm{~kg} / 33 \mathrm{dec}$ & M.R. Food and Protein Industries & $70 / 400 \mathrm{~g}$ \\
\hline Best oxygen & Sodium percarbonate & 250-500 g/acre & Univet Ltd. & - \\
\hline Oxy-Gold & Sodium Percarbonate & $250-500$ g/acre & Fishtech Ltd & - \\
\hline
\end{tabular}


It was found that some of the above chemicals are also used to remove hardness and poisonous gases e.g. Oxyflow and Quick oxygen. Some chemicals like Oxymax, $\mathrm{O}_{2}$-Marrine and Oxy plus also help in preventing diseases in fish.

\section{Chemicals used for disease treatment}

Apart from antibiotics a handful of chemicals mostly traditional were seen available in the shops for treating fish disease. No information leaflet was available on dose and methods of application of such chemicals. No pharmaceutical companies are dealing with the basic traditional chemical rather they are engaged in producing new products. These types of chemical are shown in Table 4.

Table 4. Chemicals used for disease treatment

\begin{tabular}{|l|l|l|c|}
\hline \multicolumn{1}{|c|}{ Trade Name } & \multicolumn{1}{|c|}{ Active ingredient } & \multicolumn{1}{c|}{ Source } & Price (Tk.) \\
\hline Potash & $\mathrm{KMnO}_{4}$ & Chemical seller & $185 / \mathrm{kg}$ \\
\hline Eco- solution & Eco-Solution & Chemical seller & $85 / \mathrm{L}$ \\
\hline Lime & $\mathrm{CaO}, \mathrm{Ca}(\mathrm{OH})_{2}$ & Chemical seller & $12 / \mathrm{kg}$ \\
\hline Formalin & $40 \% \mathrm{HCHO}$ & Chemical seller & $70 / \mathrm{kg}$ \\
\hline Salt & $\mathrm{NaCl}$ & Chemical seller & $8 / \mathrm{kg}$ \\
\hline Albez & $\begin{array}{l}\text { Doxycyclin, colistine sulphate }+ \text { vitamin premix } \\
+ \text { mineral }\end{array}$ & Syngenta & $253 / 250 \mathrm{~g}$ \\
\hline Protacide & $\begin{array}{l}\mathrm{C}_{22} \mathrm{H}_{42} \mathrm{CIN}+\mathrm{C}_{23} \mathrm{H}_{25} \mathrm{CIN}_{2}+\mathrm{C}_{2} \mathrm{H}_{5} \mathrm{OH}+\text { Some inert } \\
\text { component }\end{array}$ & Ellwellas & - \\
\hline Methylene Blue & $\mathrm{C}_{10} \mathrm{H}_{18} \mathrm{CIN}{ }_{3} \mathrm{~S}_{x} \mathrm{H}_{2} \mathrm{O}$ & Chemical seller & $350 / 25 \mathrm{~g}$ \\
\hline Malachite green & $\mathrm{C}_{2} \mathrm{H}_{2} \mathrm{O}_{4}$ & Chemical seller & $500 / 25 \mathrm{~g}$ \\
\hline Melathion & Active melathion & Chemical seller & $60 / 100 \mathrm{ml}$ \\
\hline Spa & Protein, chloesteren, Ca, Vitamin-D, Carotinaid & Syngenta & $4100 / \mathrm{kg}$ \\
\hline Timsen & $\begin{array}{l}\text { n-alkyl dimethyl benzyl ammonium chloride+ } \\
\text { stabilized urea }\end{array}$ & $\begin{array}{l}\text { Eon Animal Health } \\
\text { Products Ltd. }\end{array}$ & $238 / 50 \mathrm{~g}$ \\
\hline Sumithion & Fanitrothion & Chemical seller & $90 / 100 \mathrm{ml}$ \\
\hline
\end{tabular}

From above chemicals in Table 4, Eco-solution is effective for viral diseases. Melathion, Dipterex, Melachite green, Formalin, Salt, Methylin blue, etc. are useful for eradication of external parasites as well as fungal diseases. Lime is also used for common fish diseases. Spa is effective for both disease treatment and growth promotion purposes. Moreover, timsen is used for treatment of several diseases and as a disinfectant.

\section{Antibiotics for disease treatment}

In the present investigation about 14 branded antibiotics with different trade names were seen in the market as well as used by the fish farmers which are shown in Table 5. The active ingredients of such antibiotics are mainly Oxytetracycline, Chloro-tetracyclin, Amoxicillin, Co-trimoxa zole, Sulphadiazine and Sulphamethoxazole. The price seems quite affordable by the commercial aqua farmers. 
Table 5. Antibiotics for disease treatment

\begin{tabular}{|c|c|c|c|c|}
\hline Trade name & Active ingredient & Dose & Source & Price (Tk.) \\
\hline Oxysentin $20 \%$ & Oxytetracline $\mathrm{HCl} \mathrm{BP}$ & $\begin{array}{l}100-200 \mathrm{~g} / 100 \mathrm{~kg} \text { feed, } 5-7 \text { days } \\
\text { (For treatment) }\end{array}$ & $\begin{array}{l}\text { Novartis } \\
\text { Pharmaceuticals }\end{array}$ & $700 / \mathrm{kg}$ \\
\hline Chlorsteclin & Chlortetracycline & $200-300 \mathrm{~g} / 100 \mathrm{Kg}$ feed (5-7days) & $\begin{array}{l}\text { Novartis } \\
\text { Pharmaceuticals }\end{array}$ & $300 / \mathrm{kg}$ \\
\hline Ranamox & Amoxicillin Trihydrate & $\begin{array}{l}28-40 \mathrm{~g} / 100 \mathrm{bd} \text { of fish, } 10 \text { days } \\
\text { continuously. }\end{array}$ & $\begin{array}{l}\text { Renata } \\
\text { Pharmaceuticals Ltd }\end{array}$ & $140 / 100 \mathrm{~g}$ \\
\hline Renamycin & Oxytetracycline & $28-42$ g/100 kg feed, 10 days. & $\begin{array}{l}\text { Renata } \\
\text { Pharmaceuticals }\end{array}$ & $82 / 100 \mathrm{~g}$ \\
\hline Fish cure & Chloro-tetracyclin $\mathrm{HCl}$ & $500 \mathrm{~g} / 100 \mathrm{~kg}$ feed (3-5 days) & Rals Agro Ltd. & $275 / \mathrm{kg}$ \\
\hline Orgamycin $15 \%$ & $\begin{array}{l}\text { Oxytracycline } \mathrm{HCl} \mathrm{BP} \\
\text { (WSP) }\end{array}$ & $\begin{array}{l}\text { In case of prevention } 60 \mathrm{gm} / 100 \\
\mathrm{~kg} \text { feed } 10 \text { days } \\
\text { In treatment } 120-240 \mathrm{~g} / 100 \mathrm{~kg} \\
\text { feed, } 5-7 \text { days }\end{array}$ & $\begin{array}{l}\text { Organic } \\
\text { Pharmaceuticals Ltd. }\end{array}$ & $70 / 100 \mathrm{~g}$ \\
\hline $\begin{array}{l}\text { Orgacycline- } \\
15 \%\end{array}$ & Chlorotetracycline & $200-300 \mathrm{~g} / 100 \mathrm{~kg}$ feed (5-7days) & $\begin{array}{l}\text { Organic } \\
\text { Pharmaceuticals Ltd }\end{array}$ & - \\
\hline $\begin{array}{l}\text { Acimox(vet) } \\
\text { Powder }\end{array}$ & $\begin{array}{l}\text { Amoxiciline } \\
\text { (Trihydrate) }\end{array}$ & $\begin{array}{l}1 \mathrm{~g} / 1 \mathrm{~kg} \\
\text { feed }\end{array}$ & $\mathrm{ACl}$ Animal Health & $75 / 100 \mathrm{~g}$ \\
\hline Bactitab & Oxytetracyclin $20 \%$ & $50 \mathrm{~g} / \mathrm{kg}$ body weight , 5-7 days & ACl Animal Health & $70-80 / 100 \mathrm{~g}$ \\
\hline $\begin{array}{l}\text { Contrim vet } \\
\text { Bolus }\end{array}$ & Co-Trimoxa zole & $\begin{array}{l}\text { Mixed with feed; 1bolus/ 10-12 kg } \\
\text { body wt }\end{array}$ & $\begin{array}{l}\text { Square } \\
\text { Pharmaceuticals }\end{array}$ & $64 / 100 \mathrm{~g}$ \\
\hline $\begin{array}{l}\text { Otetra vet power } \\
50\end{array}$ & Oxytetracycline & $\begin{array}{l}\text { Mixed with feed; } 11-16 \mathrm{~g} / 100 \mathrm{~kg} \\
\text { body wt }\end{array}$ & $\begin{array}{l}\text { Square } \\
\text { Pharmaceuticals }\end{array}$ & $156 / 100 \mathrm{~g}$ \\
\hline Sulfatrim & $\begin{array}{l}\text { Sulphadiazine \& } \\
\text { Trimethoprim } \\
\end{array}$ & $50 \mathrm{~g} / \mathrm{kg}$ body weight , $5-7$ days & $\begin{array}{l}\text { Square } \\
\text { Pharmaceuticals }\end{array}$ & $70-80 / 100 \mathrm{~g}$ \\
\hline Oxin WS & $\begin{array}{l}\text { Oxytetracycline } \\
20 \%\end{array}$ & $50 \mathrm{mg} / \mathrm{kg}$ body weight & Navana & $70-80 / 100 \mathrm{~g}$ \\
\hline Cotrim-Vet & $\begin{array}{l}\text { Sulphamethoxazole + } \\
\text { Trimethoprime }\end{array}$ & $0.50 \mathrm{mg} / \mathrm{kg}$ body weight & $\begin{array}{l}\text { Square } \\
\text { Pharmaceuticals }\end{array}$ & $70-80 / 100 \mathrm{~g}$ \\
\hline
\end{tabular}

According to the information leaflet, all of these antibiotics are effective against bacterial. disease. According to the leaflet, Oxysentin 20\%, Orgamycin $15 \%$ and Orgacycline-15\% are also effective against EUS. It was also mentioned that Chlorsteclin and Fish cure plays vital role in growth promotion as well as effective against some disease like dropsy, tail and fin rot, gill rot of fish etc.

\section{Chemicals used as growth promoter}

Chemicals found in the chemical shops which are used as growth promoter as well as to increase production included Megavit Aqua, Aqua Boost, Aqua Savor, Vitamin premix, Fibosoel, Grow fast, Orgavit auqa, AQ-Cell , AQGrow-G, Fish vita plus, AQ Grow-L, Growmax, Nature Aqua GP, Vitamix, F Aqua , ACmix and many more. Aqua boost contains ß-glucan, immunostimulant, which enhance non-specific immunity in fish.

\section{Widely used traditional chemicals in freshwater aquaculture for health management}

Data from 50 freshwater fish farmers were analyzed to know the status of use of commonly available traditional chemicals which are shown in Table 6. 
Faruk et al.

Table 6. Widely used traditional chemicals in freshwater aquaculture

\begin{tabular}{|c|c|c|}
\hline Chemicals & $\begin{array}{c}\text { Number of respondents } \\
(\mathrm{n}=50)\end{array}$ & \% of farmer \\
\hline Lime & 40 & 80 \\
\hline Salt & 30 & 60 \\
\hline Potash & 20 & 40 \\
\hline Melathion & 10 & 20 \\
\hline
\end{tabular}

Some previous studies also revealed the similar outcome regarding the use of chemicals in aquaculture in Bangladesh (Phillips, 1996; Brown and Brooks, 2002; DoF, 2002 and Faruk et al., 2005).

\section{Widely used new chemicals in freshwater aquaculture}

From the list of a number of new chemicals found in market an attempt was made to find widely used new products by farmers. It was found that Geotox, JVzeolite, Green Zeolite, Megavit Aqua, Fish Vitaplus, AQ Grow-G, Oxy flow, Oxy max, $\mathrm{O}_{2}$-Marine were the most widely used new chemicals in freshwater aquaculture in the study area (Table 7).

Table 7. Widely used new chemicals in freshwater aquaculture

\begin{tabular}{|l|c|c|}
\hline Chemicals & Number of farmers $(\mathrm{n}=50)$ & \% of farmer \\
\hline Geotox & 12 & 24 \\
\hline JVzeolite & 14 & 28 \\
\hline Green Zeolite & 18 & 36 \\
\hline Megavit Aqua & 14 & 28 \\
\hline Fish Vitaplus & 13 & 26 \\
\hline AQ Grow-G & 12 & 24 \\
\hline Oxy flow & 11 & 22 \\
\hline Oxy max & 14 & 28 \\
\hline O $_{2}$-Marine & 18 & 36 \\
\hline
\end{tabular}

Widely used antibiotics in freshwater aquaculture

The use of antibiotic substances is the cause of much controversy. Bacterial diseases of fish can be successfully treated with antibiotics. It is widely recognized that excessive use of antibiotics contributes the development of resistant strains of bacteria (Inglis, 1996). Of the antibiotics four antibiotics like Oxysentin 20\%, Renamox, Renamycin and Orgamycine15\% were used widely by the farmers (Table 8) for the treatment of fish diseases.

Table 8. Widely used antibiotics in freshwater aquaculture

\begin{tabular}{|l|c|c|}
\hline \multicolumn{1}{|c|}{ Chemicals } & Number of farmers $(\mathrm{n}=50)$ & $\%$ of farmer \\
\hline Oxysentin $20 \%$ & 17 & 34 \\
\hline Renamox & 6 & 12 \\
\hline Renamycin & 14 & 28 \\
\hline Orgamycine $15 \%$ & 11 & 22 \\
\hline
\end{tabular}




\section{Chemical producing pharmaceutical companies}

In the present study, about 33 companies were found either producing or marketing products targeting aquaculture. ACl Animal Health Ltd., Organic Pharmaceuticals Ltd., Renata Ltd., Square Pharmaceuticals Ltd., Acme Laboratories, Novartis Animal Health Ltd etc. were seen to produce different products for aquaculture. While Eon Animal Health Products Ltd., CP Company, Rals Agro Ltd., Ellwellas Marketing Ltd. have been marketing various products from different countries including India, USA, Thailand, Taiwan, Indonesia, Malaysia and Spain. They provide detail information about the purposes, dosages, duration and method of application of chemicals in the leaflet. However, farmers had different opinion about the use and efficacy of many of the products. Some companies were seen to provide technical assistance to the farmers. Thus, the farmers fetch considerable pressure from the commercial companies to use a variety of products in their farms. Several products have been sold without any explanation of their action. The present survey reveled that among the 33 companies more than $50 \%$ of the products were produced by seven companies like Organic Pharmaceuticals Ltd. (11.32\%), Rals Agro Ltd. (9.43\%), Novartis Animal Health Ltd. (5.66\%), Eon animal Health Product Ltd. (7.55\%), ACl Animal Health Ltd. (11.32\%), CP Aquaculture (5.66\%), Square Pharmaceuticals Ltd. (5.66\%), and the rest $(43.40 \%)$ were supplied by the 26 companies (Table 9 ).

Table 9. Supply of Aquatic Chemicals (\%) producing pharmaceutical companies

\begin{tabular}{|l|c|c|}
\hline Name of Company & Number of products $\mathrm{n}=106$ & Product (\%) \\
\hline Organic & 12 & 11.32 \\
\hline Rals Agro & 10 & 9.43 \\
\hline Novartis & 6 & 5.66 \\
\hline Eon & 8 & 7.55 \\
\hline $\mathrm{ACl}$ & 12 & 11.32 \\
\hline $\mathrm{CP}$ & 6 & 5.66 \\
\hline Square & 6 & 5.66 \\
\hline Other 26 Companies. & 46 & 43.40 \\
\hline
\end{tabular}

It was also found from the present survey that the farmers were using most of the chemicals indiscriminately. There is no regulation on the use of chemicals in aquaculture in Bangladesh. On a worldwide basis, only a small number of chemicals are legally approved for use in aquaculture. The use of drugs and chemicals by the US aquaculture industry is strictly regulated by the US Food and Drug Administration (FDA) and the US Environmental Protection Agency (EPA). In the present investigation, some chemicals were found with only trade names. The sellers or farmers or extension workers do not have clear idea about the active ingredient of that particular chemical although they are using those without hesitation.

\section{Problems in use of chemicals and antibiotics}

The present study identified some problems associated with the use of chemicals and antibiotics which included i) lack of technical knowledge of fish farmers on fish health management ii) lack of knowledge about use of chemicals iii) unavailability of appropriates therapeutic iv) lack of knowledge of application of chemicals and antibiotics v)indiscriminate 
use of chemicals vi) pressure on farmers from pharmaceutical companies and pesticide sellers vii) poor quality chemicals viii) lack of assistance from GO and NGOs ix) lack of awareness about the safety issues in using hazardous chemicals $x$ ) lack of information on the label of chemical about possible hazard xi) lack of knowledge about residual effect and expiry date xii) lack of diagnostic facilities for proper disease diagnosis and xiii) lack of trained manpower to prescribe fish medicine.

\section{Conclusion}

The present study demonstrated current status of chemicals and antibiotics used in aquatic animal health management and pointed out some problems of use of these chemicals by the farmers. Simply less use of chemicals is the best alternative to minimize the adverse effects of chemicals in aquaculture. Other alternatives could be used as bioremediation and use of probiotics, immunostimulants, vaccination and alternative therapeutic. However, policy makers, researchers, and scientists should work together in addressing the issues of chemical use in aquaculture with the view to reduce the negative impacts.

\section{Acknowledgements}

The authors are grateful to the University Grants Commission (UGC) of Bangladesh for providing financial support to the project through BAURES. The authors are also thankful to all the aqua farmers, chemical retailers and medical promotion officers of different pharmaceutical companies who participated in the survey and PRA activities.

\section{References}

BFRI, 1999. Fish diseases: prevention and control. Bangladesh Fisheries Research Institute, Circular leaflet no 6. $2^{\text {nd }}$ edition. $7 \mathrm{pp}$.

Brown, D. and Brooks, A. 2002. A survey of disease impact and awareness in pond aquaculture in Bangladesh, the Fisheries and Training Extension Project- Phase 11. In: Primary Aquatic Animal Health Care in Rural, SmallScale and Aquaculture Development. Arther, J. R., M.J. Phillips, R.P. Subasinghe, M.B. Reantaso and I.H. MacRae. (eds.). FAO Fish. Tech. Pap. No. 406. pp. 85-93.

DoF, 2002. Fish Fortnight Compendium. 10-24 August 2002, Department of Fisheries, Matsha Bhaban, Dhaka. pp. 44-45.

Faruk, M.A.R., Alam, M.J., Sarker, M.M.R. and M.B. Kabir, 2004. Status of fish disease and health management practices in rural freshwater aquaculture of Bangladesh. Pakistan J. Biol. Sci., 7 (12): 2092-2098.

Faruk, M.A.R., Sultana, N. and M.B. Kabir, 2005. Use of chemicals in aquaculture activities in Mymensingh area, Bangladesh. Bangladesh J. Fish. 29 (1-2): 1-10.

GESAMP (Joint Group of Experts on the Scientific Aspects of Marine Environmental Protection). 1997. Towards safe and effective use of chemicals in coastal aquaculture. Rep. Stud. (IMO/ FAO/ UNESCO/ IOC// WMO/ WHO/ IAEA/ UN/ UNEP, 65: $40 \mathrm{pp}$.

Hasan, M.R. and Ahmed, G.U. 2002. Issues in carp hatcheries and nurseries in Bangladesh, with special reference to health management. In: Primary Aquatic Animal Health Care in Rural, Small-Scale. Arthur, J. R., M. J. Phillips, R. P. Subasinghe, M. B. Reantaso and 1. H. MacRae (eds.). Aquaculture Development. FAO Fish. Tech. Pap. No. 406. pp. 147-164.

Inglis, V., 1996. Antibacterial chemotherapy in aquaculture: review of practice, associated risks and need for action. In: Use of Chemicals in Aquaculture in Asia. Arthur, J.R., C.R. lavilla-Pitogo and R.P. Subasinghe (eds.). Southeast Asian Fisheries Development Centre, Aquaculture Department Tigbauan, Iloilo, Philippines. pp.7-22. 
Karim, M. and Stellwagen, J. 1998. Final Report on Fourth Fisheries Projects: shrimp aquaculture (Preparatory phase for National development Program). Department of Fisheries, Ministry of Fisheries \& Livestock, Bangladesh.

Phillips, M. 1996. The use of chemicals in carp and shrimp aquaculture in Bangladesh, Cambodia, Lao PDR, Nepal, Pakistan, Sri Lanka and Viet Nam. In: Use of Chemicals in Aquaculture in Asia. Arthur, J.R., C.R. LavillaPitogo, R. P. Subasinghe (eds). Southeast Asian Fisheries Development Center, Aquaculture Department Tigbauan, Iloilo, Philippines. pp 75-84.

Plumb, J.A. 1992. Disease control in aquaculture. In: Disease in Asian Aquaculture (edited by I.M. Shariff, R.P. Subasinghe \& J. R. Arthur) Fish Health Section of the Asian Fisheries Society, Manila, Philippines, pp. 3-17.

Subasinghe, R.P., Barg U. and Tacon, A. 1996. Chemicals in Asian aquaculture: need, usage, issues and challenges. In: Use of Chemicals in Aquaculture in Asia. Arthur, J.R., C.R. Lavilla-Pitogo, R. P. Subasinghe (eds). Southeast Asian Fisheries Development Center, Aquaculture Department Tigbauan, Iloilo, Philippines. pp 1-6. 